COMMISSIONS G1 AND G4 OF THE IAU

INFORMATION BULLETIN ON VARIABLE STARS

Volume 62 Number 6195 DOI: 10.22444/IBVS.6195

Konkoly Observatory

Budapest

20 January 2017

HU ISSN $0374-0676$

\title{
CCD MINIMA FOR SELECTED ECLIPSING BINARIES IN 2016
}

NELSON, R. H.

1393 Garvin Street, Prince George, BC, Canada, V2M $3 Z 1$ e-mail: bob.nelson@shaw.ca

Observatory and telescope:

Sylvester Robotic Observatory (SyRO): $33 \mathrm{~cm} \mathrm{f/4.5} \mathrm{Newtonian} \mathrm{on} \mathrm{a} \mathrm{Paramount}$ $\mathrm{ME}$

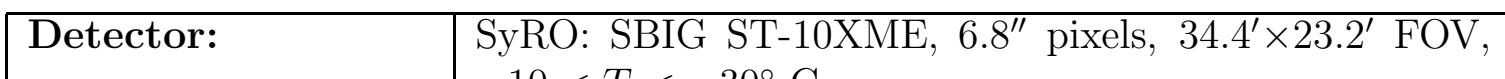
$-10<T<-30^{\circ} \mathrm{C}$

\section{Method of data reduction:}

Bias and dark subtraction, flat-fielding using light-box flats; aperture photometryall using MIRA, by Mirametrics. Check stars were used throughout.

\section{Method of minimum determination:}

Digital tracing paper method, bisection of chords, curve fitting, and (occasionally) Kwee and van Woerden (1956) 


\begin{tabular}{|c|c|c|c|c|c|}
\hline \multicolumn{6}{|c|}{ Times of minima: } \\
\hline Star name & $\begin{array}{l}\text { Time of min. } \\
\text { HJD } 2400000+\end{array}$ & Error & Type & Filter & $\begin{array}{ll}O-C & \text { Rem. } \\
{[\text { day }]} & \end{array}$ \\
\hline QX And & 57734.6099 & 0.0002 & $\mathrm{I}$ & $\mathrm{c}$ & 0.0016 \\
\hline V0527 And & 57654.7928 & 0.0003 & I & $\mathrm{c}$ & 0.0020 \\
\hline V1747 Aql & 57521.9096 & 0.0003 & $\mathrm{I}$ & $\mathrm{c}$ & 0.0023 \\
\hline SS Ari & 57699.6733 & 0.0003 & I & $\mathrm{R}$ & -0.0061 \\
\hline BN Ari & 57702.6294 & 0.0003 & $\mathrm{I}$ & VRI & -0.0003 \\
\hline BN Ari & 57707.5698 & 0.0007 & II & VRI & 0.0006 \\
\hline BN Ari & 57707.7187 & 0.0002 & $\mathrm{I}$ & VRI & -0.0002 \\
\hline BN Ari & 57728.6745 & 0.0005 & $\mathrm{I}$ & VRI & -0.0001 \\
\hline BN Ari & 57729.5727 & 0.0003 & $\mathrm{I}$ & VRI & -0.0000 \\
\hline BN Ari & 57730.621 & 0.0002 & II & VRI & 0.0005 \\
\hline V0410 Aur & 57738.5905 & 0.0002 & $\mathrm{I}$ & $\mathrm{R}$ & 0.0000 \\
\hline V0644 Aur & 57734.74 & 0.0002 & $\mathrm{I}$ & c & -0.0001 \\
\hline TY Boo & 57477.829 & 0.0002 & I & c & -0.0006 \\
\hline TZ Boo & 57439.8916 & 0.0002 & I & $\mathrm{R}$ & 0.0007 \\
\hline TZ Boo & 57753.1011 & 0.0003 & II & $\mathrm{R}$ & 0.0020 \\
\hline CK Boo & 57498.7485 & 0.0008 & I & $\mathrm{c}$ & -0.0047 \\
\hline GI Boo & 57514.7897 & 0.0002 & $\mathrm{I}$ & c & 0.0033 \\
\hline GN Boo & 57476.8355 & 0.0001 & I & $\mathrm{c}$ & -0.0059 \\
\hline GQ Boo & 57439.988 & 0.0004 & II & $\mathrm{c}$ & -0.0019 \\
\hline GQ Boo & 57498.8367 & 0.0002 & II & c & -0.0030 \\
\hline GR Boo & 57465.8222 & 0.0003 & II & $\mathrm{c}$ & -0.0005 \\
\hline GR Boo & 57492.7537 & 0.0003 & I & $\mathrm{c}$ & -0.0008 \\
\hline GS Boo & 57463.8021 & 0.0003 & II & c & 0.0009 \\
\hline HН Boo & 57487.7804 & 0.0002 & I & $\mathrm{c}$ & -0.0024 \\
\hline HН Boo & 57499.7308 & 0.0002 & II & $\mathrm{c}$ & -0.0019 \\
\hline IK Boo & 57497.7395 & 0.0002 & I & $\mathrm{c}$ & -0.0079 \\
\hline QT Boo & 57493.788 & 0.002 & I & c & -0.0035 \\
\hline $\mathrm{AO}$ Cam & 57672.798 & 0.0002 & II & c & -0.0008 \\
\hline CV Cam & 57747.622 & 0.0002 & II & $\mathrm{R}$ & 0.0003 \\
\hline LR Cam & 57671.9582 & 0.0001 & I & $\mathrm{R}$ & 0.0048 \\
\hline NR Cam & 57707.8518 & 0.0003 & II & $\mathrm{c}$ & -0.0006 \\
\hline NX Cam & 57739.6351 & 0.0006 & II & $\mathrm{c}$ & 0.0004 \\
\hline V0337 Cam & 57736.5865 & 0.0003 & I & $\mathrm{R}$ & -0.0005 \\
\hline V0383 Cam & 57625.9666 & 0.0003 & II & c & -0.0002 \\
\hline V0403 Cam & 57737.7574 & 0.0003 & II & $\mathrm{c}$ & 0.0014 \\
\hline V0474 Cam & 57729.8173 & 0.0003 & II & $\mathrm{c}$ & -0.0008 \\
\hline G3715-0043 Cam & 57739.6261 & 0.0006 & 0 & $\mathrm{c}$ & 0.0000 \\
\hline TW Cas & 57619.9773 & 0.0002 & $\mathrm{I}$ & VRI & 0.0020 \\
\hline ZZ Cas & 57617.8966 & 0.0001 & I & $\mathrm{c}$ & 0.0025 \\
\hline BS Cas & 57615.8603 & 0.0001 & I & $\mathrm{c}$ & 0.0001 \\
\hline EY Cas & 57637.8343 & 0.0002 & I & $\mathrm{c}$ & -0.0028 \\
\hline IR Cas & 57672.6806 & 0.0002 & I & VRI & -0.0002 \\
\hline IR Cas & 57673.7035 & 0.0002 & II & VRI & 0.0017 \\
\hline V0375 Cas & 57625.8179 & 0.0001 & $\mathrm{I}$ & $\mathrm{R}$ & -0.0034 \\
\hline V1063 Cas & 57645.8468 & 0.0002 & II & c & 0.0004 \\
\hline V1139 Cas & 57483.7239 & 0.0002 & II & c & 0.0005 \\
\hline V0736 Cep & 57619.7376 & 0.0006 & $\mathrm{I}$ & VRI & -0.0004 \\
\hline G4500-0730 Cер & 57661.635 & 0.0002 & II & $\mathrm{c}$ & 0.0001 \\
\hline
\end{tabular}




\begin{tabular}{|c|c|c|c|c|c|}
\hline \multicolumn{6}{|c|}{ Times of minima: } \\
\hline Star name & $\begin{array}{l}\text { Time of min. } \\
\text { HJD 2400000+ }\end{array}$ & Error & Type & Filter & $\begin{array}{ll}O-C & \text { Rem. } \\
\text { [day] } & \end{array}$ \\
\hline$\overline{\mathrm{BX}} \mathrm{CMi}$ & 57425.6877 & 0.0002 & $\bar{I}$ & $\bar{R}$ & 0.0005 \\
\hline DS CMi & 57441.6687 & 0.0005 & I & $\mathrm{c}$ & 0.0003 \\
\hline EH Cnc & 57730.9149 & 0.0001 & II & $\mathrm{c}$ & 0.0018 \\
\hline IU Cnc & 57463.6988 & 0.0002 & $\mathrm{I}$ & c & 0.0051 \\
\hline G1936-0040 Cnc & 57738.8988 & 0.0002 & II & $\mathrm{c}$ & 0.0002 \\
\hline UX CVn & 57454.7799 & 0.0003 & $\mathrm{I}$ & c & -0.0012 \\
\hline $\mathrm{DH} \mathrm{CVn}$ & 57723.9964 & 0.0006 & II & c & -0.0001 \\
\hline DR CVn & 57480.7116 & 0.0005 & II & VRI & -0.0074 \\
\hline DX CVn & 57443.9182 & 0.0002 & II & $\mathrm{c}$ & 0.0008 \\
\hline EL CVn & 57476.713 & 0.002 & I & $\mathrm{R}$ & -0.0006 \\
\hline FV CVn & 57433.9306 & 0.0003 & II & c & -0.0002 \\
\hline G2530-1069 CVn & 57747.0468 & 0.0002 & $\mathrm{I}$ & $\mathrm{c}$ & 0.0007 \\
\hline RW Com & 57425.8104 & 0.0004 & $\mathrm{I}$ & $\mathrm{c}$ & -0.0008 \\
\hline RZ Com & 57478.7858 & 0.0001 & I & $\mathrm{R}$ & 0.0046 \\
\hline SS Com & 57479.7958 & 0.0003 & II & $\mathrm{c}$ & 0.0002 \\
\hline CM Com & 57477.722 & 0.0003 & $\mathrm{I}$ & c & -0.0018 \\
\hline AS CrB & 57441.9686 & 0.0002 & II & $\mathrm{c}$ & 0.0044 \\
\hline $\mathrm{AV} \mathrm{CrB}$ & 57478.8942 & 0.0001 & $\mathrm{I}$ & $\mathrm{c}$ & -0.0020 \\
\hline V0401 Cyg & 57487.9323 & 0.0002 & $\mathrm{I}$ & $\mathrm{c}$ & -0.0070 \\
\hline V0687 Cyg & 57510.9305 & 0.0001 & $\mathrm{I}$ & $\mathrm{c}$ & -0.0030 \\
\hline V0859 Cyg & 57530.8584 & 0.0002 & II & $\mathrm{c}$ & 0.0009 \\
\hline V2197 Cyg & 57514.918 & 0.0001 & $\mathrm{I}$ & $\mathrm{c}$ & -0.0008 \\
\hline V2282 Cyg & 57510.839 & 0.0002 & II & $\mathrm{c}$ & -0.0000 \\
\hline V2364 Cyg & 57498.9433 & 0.0006 & $\mathrm{I}$ & $\mathrm{c}$ & -0.0016 \\
\hline V2477 Cyg & 57499.9633 & 0.0001 & I & $\mathrm{R}$ & -0.0001 \\
\hline BV Dra & 57463.9201 & 0.0003 & I & $\mathrm{V}$ & -0.0005 \\
\hline BW Dra & 57463.8751 & 0.0005 & II & $\mathrm{V}$ & 0.0019 \\
\hline EF Dra & 57515.7979 & 0.0005 & I & $\mathrm{c}$ & 0.0025 \\
\hline GQ Dra & 57483.8383 & 0.0002 & I & $\mathrm{V}$ & 0.0006 \\
\hline IV Dra & 57492.8669 & 0.0004 & II & $\mathrm{c}$ & 0.0056 \\
\hline V0415 Dra & 57492.9764 & 0.0004 & I & $\mathrm{c}$ & -0.0030 \\
\hline G3870-1172 Dra & 57448.8752 & 0.0002 & II & $\mathrm{c}$ & 0.0009 \\
\hline G3870-1172 Dra & 57497.8737 & 0.0001 & II & $\mathrm{R}$ & 0.0015 \\
\hline G3881-0579 Dra & 57465.9382 & 0.0001 & II & c & 0.0007 \\
\hline G3929-1500 Dra & 57522.817 & 0.0002 & II & $\mathrm{c}$ & -0.0013 \\
\hline G4215-1480 Dra & 57480.8837 & 0.0002 & $\mathrm{I}$ & $\mathrm{c}$ & 0.0000 \\
\hline G4439-1124 Dra & 57499.8687 & 0.0004 & $\mathrm{I}$ & $\mathrm{c}$ & 0.0055 \\
\hline G4439-1124 Dra & 57516.7866 & 0.0008 & II & $\mathrm{c}$ & 0.0011 \\
\hline V0415 Gem & 57734.8607 & 0.0004 & II & $\mathrm{c}$ & -0.0004 \\
\hline G1886-1869 Gem & 57738.765 & 0.0005 & I & $\mathrm{c}$ & -0.0007 \\
\hline V1036 Her & 57521.8043 & 0.0002 & II & c & 0.0018 \\
\hline V1067 Her & 57479.9096 & 0.0002 & II & $\mathrm{c}$ & -0.0001 \\
\hline V1103 Her & 57475.863 & 0.0003 & I & $\mathrm{c}$ & -0.0006 \\
\hline V1167 Her & 57475.9724 & 0.0004 & $\mathrm{I}$ & $\mathrm{R}$ & 0.0027 \\
\hline V1198 Her & 57524.8347 & 0.0003 & I & $\mathrm{c}$ & 0.0026 \\
\hline V1284 Her & 57513.8723 & 0.0001 & $\mathrm{I}$ & $\mathrm{c}$ & 0.0001 \\
\hline V1286 Her & 57493.8911 & 0.0002 & I & $\mathrm{c}$ & -0.0050 \\
\hline V1289 Her & 57511.9014 & 0.0001 & $\mathrm{I}$ & c & -0.0005 \\
\hline
\end{tabular}




\begin{tabular}{|c|c|c|c|c|c|}
\hline \multicolumn{6}{|c|}{ Times of minima: } \\
\hline Star name & $\begin{array}{l}\text { Time of min. } \\
\text { HJD } 2400000+\end{array}$ & Error & Type & Filter & $\begin{array}{ll}O-C & \text { Rem. } \\
\text { [day }] & \end{array}$ \\
\hline V1302 Her & 57522.912 & 0.0003 & $\mathrm{I}$ & $\mathrm{c}$ & 0.0010 \\
\hline V1333 Her & 57481.9646 & 0.0001 & II & $\mathrm{c}$ & 0.0001 \\
\hline SW Lac & 57674.6566 & 0.0002 & $\mathrm{I}$ & $\mathrm{R}$ & 0.0022 \\
\hline UZ Leo & 57731.003 & 0.0001 & II & $\mathrm{V}$ & 0.0032 \\
\hline ET Leo & 57738.9741 & 0.0003 & II & $\mathrm{R}$ & 0.0005 \\
\hline XX LMi & 57454.6765 & 0.0003 & I & $\mathrm{c}$ & -0.0019 \\
\hline AG LMi & 57442.6695 & 0.0002 & I & $\mathrm{c}$ & 0.0001 \\
\hline SW Lyn & 57735.8821 & 0.0002 & I & c & 0.0031 \\
\hline CL Lyn & 57735.7652 & 0.0003 & $\mathrm{I}$ & c & -0.0007 \\
\hline DE Lyn & 57728.7772 & 0.0003 & II & c & -0.0019 \\
\hline FO Lyn & 57728.9316 & 0.0002 & II & $\mathrm{c}$ & 0.0001 \\
\hline PY Lyr & 57508.964 & 0.002 & I & $\mathrm{c}$ & 0.0000 \\
\hline V0563 Lyr & 57481.8633 & 0.0002 & I & c & -0.0004 \\
\hline V0579 Lyr & 57477.927 & 0.001 & $\mathrm{I}$ & $\mathrm{c}$ & -0.0016 \\
\hline V0653 Lyr & 57476.9573 & 0.0001 & I & c & 0.0003 \\
\hline V0927 Mon & 57737.8363 & 0.0003 & II & $\mathrm{c}$ & 0.0000 \\
\hline V0508 Oph & 57497.965 & 0.0002 & II & $\mathrm{R}$ & 0.0030 \\
\hline V2713 Oph & 57480.9854 & 0.0003 & I & c & 0.0005 \\
\hline V0517 Ori & 57747.778 & 0.002 & I & c & 0.0004 \\
\hline V1833 Ori & 57746.7359 & 0.0005 & I & $\mathrm{c}$ & -0.0004 \\
\hline V1848 Ori & 57389.684 & 0.0002 & $\mathrm{I}$ & $\mathrm{c}$ & 0.0012 \\
\hline V2762 Ori & 57730.794 & 0.0002 & I & I & -0.0003 \\
\hline DK Peg & 57646.8229 & 0.0003 & I & c & 0.0022 \\
\hline V0523 Peg & 57637.7164 & 0.0002 & II & $\mathrm{c}$ & 0.0002 \\
\hline V0535 Peg & 57624.7678 & 0.0003 & I & $\mathrm{c}$ & 0.0047 \\
\hline V0576 Peg & 57615.7732 & 0.0002 & $\mathrm{I}$ & $\mathrm{c}$ & -0.0012 \\
\hline V0619 Peg & 57624.8949 & 0.0002 & $\mathrm{I}$ & $\mathrm{R}$ & 0.0005 \\
\hline RT Per & 57618.8972 & 0.0001 & I & $\mathrm{c}$ & 0.0014 \\
\hline RT Per & 57646.928 & 0.0001 & I & c & 0.0016 \\
\hline V0881 Per & 57626.8723 & 0.0002 & II & $\mathrm{c}$ & -0.0013 \\
\hline DZ Psc & 57653.8041 & 0.0003 & I & $\mathrm{c}$ & 0.0052 \\
\hline HL Psc & 57731.5992 & 0.0005 & II & $\mathrm{c}$ & -0.0065 \\
\hline HO Psc & 57742.5931 & 0.0004 & II & $\mathrm{c}$ & 0.0009 \\
\hline V0384 Ser & 57513.7615 & 0.0001 & I & c & -0.0025 \\
\hline AH Tau & 57635.9722 & 0.0008 & II & $\mathrm{c}$ & -0.0019 \\
\hline EQ Tau & 57737.6095 & 0.0002 & II & $\mathrm{c}$ & -0.0005 \\
\hline V0781 Tau & 57751.6673 & 0.0002 & I & $\mathrm{R}$ & -0.0016 \\
\hline V1241 Tau & 57672.867 & 0.002 & II & VRI & -0.0056 \\
\hline V1332 Tau & 57742.7253 & 0.0005 & II & $\mathrm{c}$ & -0.0013 \\
\hline XY UMa & 57427.7634 & 0.0003 & II & $\mathrm{R}$ & -0.0005 \\
\hline AA UMa & 57707.9965 & 0.0001 & I & $\mathrm{c}$ & 0.0042 \\
\hline HH UMa & 57437.744 & 0.0002 & II & $\mathrm{R}$ & 0.0014 \\
\hline LP UMa & 57453.716 & 0.0004 & I & $\mathrm{c}$ & -0.0045 \\
\hline MQ UMa & 57746.8928 & 0.0002 & I & $\mathrm{c}$ & 0.0088 \\
\hline MT UMa & 57735.9896 & 0.0005 & I & $\mathrm{c}$ & -0.0005 \\
\hline NU UMa & 57448.8067 & 0.0003 & $\mathrm{I}$ & $\mathrm{V}$ & 0.0009 \\
\hline G4386-0604 UMa & 57747.9238 & 0.0002 & $\mathrm{I}$ & $\mathrm{R}$ & -0.0041 \\
\hline KN Vul & 57516.9138 & 0.0003 & I & $\mathrm{c}$ & 0.0004 \\
\hline
\end{tabular}




\section{Remarks:}

To save space, GSC star names have been shortened to a leading "G" only; times of minimum are heliocentric Julian dates with the leading 24 removed.

$O-C$ values were computed using elements computed from the $O-C$ database listed in the references (Nelson, 2016).

\section{Acknowledgements:}

Thanks are due to Environment Canada for the website satellite views (see reference below) that were essential in predicting clear times for observing runs in this cloudy locale. Thanks are also due to Attilla Danko for his 'Clear Sky Charts', (see below). This research has made use of the SIMBAD database, operated at CDS, Strasbourg, France.

References:

Danko, A., Clear Sky Charts, http://cleardarksky.com/

Kwee, K.K., \& van Woerden, H., 1956, B.A.N. 12, 327

Nelson, R.H. 2016, Bob Nelson's O-C Files, http://www . aavso.org/bob-nelsons-o-c-files

Satellite Images for North America, http://weather.gc.ca/ 